\title{
Integration of Geriatric Assessment in the Care of Patients with Gastrointestinal Malignancies
}

\author{
Siri Rostofta,b \\ a Department of Geriatric Medicine, Oslo University Hospital, Oslo, Norway; \\ bUniversity of Oslo, Oslo, Norway
}

\section{Keywords}

Geriatric assessment - Geriatric intervention · Frailty •

Gait speed · Preoperative assessment

\section{Summary}

Background: The majority of patients with gastrointestinal (GI) malignancies are older. Recently, it has become evident that elements from a geriatric assessment (GA) are powerful predictors of outcomes such as postoperative morbidity and mortality, length of stay, type of treatment received, and survival across several GI tumor types in older adults. A GA is a systematic evaluation of functional status, comorbidities, polypharmacy, cognition, nutritional status, emotional status, and social support. Methods: A PubMed search was performed in order to identify clinical studies investigating the association between GA and outcomes in patients with GI malignancies. Results: A total of 31 studies were included in this review. For colorectal cancer, the evidence linking GA variables and frailty to negative outcomes is substantial and consistent. The data regarding other $\mathrm{GI}$ malignancies is more limited, but generally shows the same findings. Conclusion: Increasing data shows that elements from a GA and frailty are consistently associated with negative short- and long-term treatment outcomes in older patients with GI malignancies. Future studies should investigate the impact of geriatric interventions on outcomes.

\section{Introduction}

Gastrointestinal (GI) malignancies are common in older patients, and due to the heterogeneity of the older population, cancer specialists dealing with these patients need to assess the patients beyond chronological age. Over the past decades, it has become evident that elements from a geriatric assessment (GA) are powerful predictors of outcomes such as postoperative morbidity and mortality, length of stay, type of treatment received, and survival across several GI tumor types in older adults. A GA is a systematic evaluation of functional status, comorbidities, polypharmacy, cognition, nutritional status, emotional status, and social support in older adults [1]. In addition to predicting risk, the GA provides other advantages: Identification of impairments that were unknown and that influence the treatment trajectory, e.g. cognitive impairment; development of interventions to improve impairments, e.g. nutritional interventions; optimization of comorbidities; and establishment of a pre-treatment baseline of functional status in order to recognize a decline. Older patients with impairments in the domains of GA may be considered frail if the impairments are across several domains or severe in one domain, such as dementia. The term frailty generally describes an individual with increased vulnerability towards stressors such as surgery or chemotherapy [2]. The goal of this paper is to assess the existing evidence of GA in GI malignancies (table 1), and to discuss implications for clinical practice.

\section{Methods}

The aim was to identify clinical studies that investigated the association between GA and outcomes in patients with the following GI malignancies: colorectal cancer (CRC), gastric cancer, esophageal cancer, liver cancer, biliary cancer, and pancreatic cancer. The following search was performed on March 3, 2017 in PubMed:

\section{KARGER}

() 2017 S. Karger GmbH, Freiburg

Fax +497614520714
Dr. Siri Rostoft 
Table 1. Geriatric Assessment - examples of domains and tests

\begin{tabular}{|c|c|c|c|}
\hline Domain & Test (example) & Score & Interpretation \\
\hline \multirow[t]{2}{*}{ Functional status - ADL } & Barthel's ADL index [3]; & $0-20$ & \multirow[t]{2}{*}{ higher score indicates better functioning } \\
\hline & Nottingham Extended ADL index [4] & $0-66$ & \\
\hline \multirow{3}{*}{$\begin{array}{l}\text { Functional status - } \\
\text { objective physical } \\
\text { performance measures }\end{array}$} & gait speed [5]; & speed m/s; & $<0.8 \mathrm{~m} / \mathrm{s}$ slow walker; \\
\hline & Timed Up and Go (TUG) [6]; & number of sec; & >19 s slow walker; \\
\hline & Short Physical Performance Battery (SPPB) [7] & $0-12$ & higher score indicates better functioning \\
\hline \multirow[t]{2}{*}{ Comorbidity } & Charlson's comorbidity index [8]; & $0-9$ & higher score - more comorbidity; \\
\hline & Cumulative Illness Rating Scale (CIRS) [9] & $\begin{array}{l}\text { number of comorbidities } \\
\text { grade } 3 \text { or } 4\end{array}$ & higher number, more severe comorbidity \\
\hline Polypharmacy & number of drugs & & \\
\hline \multirow[t]{2}{*}{ Cognitive function } & Mini-Cog ${ }^{\text {Th }}[10]$ & $0-5$ & $<3$ = cognitive impairment \\
\hline & MoCA [11] & $0-30$ & $<24=$ cognitive impairment \\
\hline Nutritional status & Mini Nutritional Assessment (MNA) [12] & $0-30$ & $<23.5$, risk of malnutrition/malnourished \\
\hline Emotional status & Geriatric Depression Scale (GDS) [13] & $0-30$ & $\begin{array}{l}>13 \text { indicates depression with high } \\
\text { sensitivity and specificity }\end{array}$ \\
\hline
\end{tabular}

(geriatric assessment AND (gastric cancer OR esophag* cancer OR biliary cancer OR liver cancer OR pancreatic cancer OR colorectal cancer)). Only papers in English were included. Studies were not eligible if the cohort consisted of non-cancer patients. Reviews and studies that did not include GA data were excluded.

\section{Results}

The search yielded 163 references. 132 studies were excluded due to reviews without original data $(n=46)$, not including GA data $(n=47)$, and not being relevant due to a mix of cancer types (GI and non-GI) or non-cancer data $(\mathrm{n}=39)$. A total of 31 studies were original studies including data on GA as well as GI cancer.

\section{Geriatric Assessment and Colorectal Cancer}

Most studies were on CRC $(n=20)$. Overall, it is evident that elements from a GA predict both short- and long-term outcomes such as postoperative complications [14-17], length of stay [14], survival [18-20], and type of treatment received [18]. Furthermore, a composite measure such as frailty is a strong predictor of negative outcomes $[14,19]$. In a registry-based study by Koroukian et al. [18], comorbidities were associated with an increased likelihood of surgery only, and both functional limitations and geriatric syndromes were associated with a decreased likelihood to undergo surgery. Two or more functional limitations were associated with overall mortality. In a prospective study of 182 patients aged 70 years and older undergoing elective CRC surgery, being frail was associated with an increased risk of postoperative complications and poor 5-year survival $[14,19]$. The domains of the GA that independently predicted poor outcomes were severe comorbidity, functional impairment, depression, and malnutrition [21]. The pa- tients were assessed 16-28 months after surgery, and many experienced a decline in functional status measured by activities of daily living (ADL) [22]. However, quality of life was not negatively affected, not even in patients that were considered frail prior to surgery [23]. Later, prospective studies from Korea, Japan, and the Netherlands have confirmed the results that GA-based frailty is a predictor of major postoperative complications in patients undergoing CRC surgery [15-17]. A prospective study in 82 patients over the age of 75 years looked at the physical frailty phenotype (weight loss, exhaustion, slow walking speed, reduced strength, low physical activity) when predicting postoperative morbidity after elective CRC resection. About $25 \%$ of the cohort was frail, and this group had a four times higher risk of developing major complications [24]. Conflicting results were found in another study looking at the physical frailty phenotype; here, only a GA-based frailty measure predicted morbidity, but the physical frailty phenotype was associated with poor survival [25]. An Italian study has confirmed that frailty predicts mortality across different measures in older patients with CRC [20]. In a registry-based study of 12,979 individuals aged 80 years and older undergoing elective colectomy for stage I-III colon cancer, the authors analyzed the 90-day mortality in relation to frailty (proxy measure from registry data), dementia, and comorbidities [26]. Older age, male gender, frailty, increased hospitalizations in the prior year, and dementia were most strongly associated with poorer survival. Interestingly, $86 \%$ of patients were alive at 1 year, but $6.6 \%$ of patients died by 90 days postoperatively, mostly of causes unrelated to their cancer diagnosis. Patient frailty was the strongest predictor of poor short-term postoperative survival, and the most common frailty criteria in this study were walking difficulties, weight loss, and frequent falls. A Dutch study retrospectively compared two cohorts of patients who underwent surgery for CRC [27]. One cohort consisting of 443 pa- 
tients was considered frail, and frail patients underwent a specialist GA followed by interventions. Frail patients who were seen by a geriatrician did not experience more complications than the comparison cohort; thus, the authors conclude that the GA and intervention have a positive influence on the postoperative outcomes. For patients with metastatic CRC, several studies have identified frailty as a predictive factor of mortality risk $[28,29]$, while malnutrition seems to predict both mortality and chemotherapy toxicity [28]. Other studies in patients with CRC found cognitive function and functional status to predict severe toxicity and unexpected hospitalizations [30]. Another study confirmed that functional status measured by instrumental ADL was predictive of overall survival [31]. A small prospective study from the USA found that the Vulnerable Elders Survey-13 (VES-13), a screening for overall functional status, was an independent predictor of survival in 38 patients with CRC receiving chemotherapy [32]. VES-13 outperformed Eastern Cooperative Oncology Group Performance Status (ECOG PS) and age. In a study of treatment delivery in older patients with CRC, the authors found that $20 \%$ of patients who were fit according to the GA did not receive standard treatment [33], and interpreted this as under-treatment. They also found that $58 \%$ of patients who were considered at risk based on the GA did receive standard treatment, with a subsequent risk of over-treatment. The GA uncovered unknown deficiencies in $40 \%$ of patients. However, even though the results of the GA were communicated to the treating physicians in this study, only a minority consulted them.

In conclusion, there is now vast evidence that elements of a GA and a composite measure of frailty predict outcomes of treatment for CRC - both for surgery and chemotherapy. In particular, all studies find that functional status is a consistent predictor of outcomes. A GA could potentially lead to an improved selection of patients for the different treatment options - in order to avoid both under-treatment of fit older patients and over-treatment of frail older patients.

\section{Geriatric Assessment and Gastroesophageal Cancer}

The evidence base is much more limited in gastroesophageal cancer. There are few studies, and most of these are retrospective and have only analyzed selected domains of the GA, thereby limiting the utility. Most authors focus on nutritional status, which is often affected in patients with these cancer types. In a retrospective cohort study of 279 patients with a median age of 64 years who underwent gastrectomy for gastric or gastroesophageal adenocarcinoma, functional status, measured by an ECOG PS $>0$, was predictive of major morbidity, readmission, and length of stay [34]. Weight loss and polypharmacy were associated with major postoperative morbidity only. Another retrospective cohort study of 180 patients operated for gastric adenocarcinoma sought to investigate whether frailty assessed by the Groningen Frailty Indicator (GFI) and nutritional status assessed by the Short Nutritional Assessment Questionnaire (SNAQ) predicted in-hospital mortality [35]. Both measures independently predicted the risk of in-hospital mortality and complications, and were stronger predictors than age and the American Society of Anesthesiologists (ASA) classification. The odds ratio for in-hospital mortality with a GFI score $\geq 3$ was 3.96 and the 95\% confidence interval 1.12-14.09. The Geriatric Nutritional Risk Index (GNRI) was found to predict respiratory complications in a cohort of 122 patients with a mean age of 64 years who underwent esophagectomy and gastric tube reconstruction due to esophageal cancer [36]. In a similar cohort in a retrospective study from Japan, patients with preoperative sarcopenia experienced more pulmonary complications than those without sarcopenia [37]. Patients in the sarcopenia group were older, had more frequent weight loss, and had higher GNRI scores. Smoking was also an independent predictor of pulmonary complications. In a prospective study from China, the occurrence of sarcopenia as a predictor of postoperative complications following total gastrectomy in patients with gastric cancer was confirmed [38]. In this study, the authors state that frailty was evaluated by sarcopenia. However, this method of defining frailty is too limited and deviates somewhat from the usual definitions of frailty in oncology [2]. The authors found that body mass index, ASA classification, and comorbidity did not predict complications independently, while nutritional risk was an independent predictor of complications. In a Japanese study in 91 patients older than 75 years who underwent esophagectomy for esophageal cancer, the association between GA variables and postoperative delirium was investigated [39]. Postoperative delirium was significantly associated with preoperative cognitive impairment and depression. Cognitive impairment is a wellknown predictor of postoperative delirium in various settings [40]. In a phase II study from 2011, 42 patients over 70 years with locally advanced or metastatic gastric cancer were treated with FOLFIRI, fluorouracil, and folic acid combined with GA [41]. Geriatric functions were not altered by the treatment, and nutritional status actually improved in some patients. The population was globally autonomous with good ADL and ECOG scores; however, over half of the patients were at risk for malnutrition or malnourished. This shows that chemotherapy treatment may improve geriatric domains in selected patients with advanced cancer, even if they are older.

\section{Geriatric Assessment and Hepatocellular Carcinoma, Pancreatic} Cancer and Hepatopancreaticobiliary Surgery

One prospective study investigated the association between GA and postoperative morbidity in 71 patients over 69 years with a diagnosis of hepatocellular carcinoma [42]. The GA consisted of two frailty screening tools - the geriatric 8 (G8) and the VES-13 - as well as depression, comorbidities, cognition, and nutrition. 18 patients (25\%) experienced postoperative complications (ClavienDindo classification II-IVb). The G8 and nutritional status were significantly associated with complications in univariate analyses, but only G8 was independently predictive after correcting for perioperative factors. In a cohort of 518 patients undergoing hepatopancreaticobiliary (HPB) surgery, the authors tried to develop a preoperative frailty risk model to predict mortality among patients 65 years and older [43]. Cancer was present in 75\% of the cohort. Preoperative GA data included comorbidity, sarcopenia, and nutritional status. Unfortunately, no data regarding functional status, 
not even ECOG status, was collected, thereby limiting the utility of the study. Factors independently associated with 1-year mortality were comorbidity, malignant disease, and sarcopenia.

In pancreatic cancer, the association between GA parameters and major postoperative complications (Clavien-Dindo > II) has been investigated in a prospective study of 76 patients older than 60 years undergoing pancreaticoduodenectomy [44]. Exhaustion measured as a part of the physical frailty phenotype predicted complications as well as length of hospital stay and intensive care admission. An objective physical performance measure, i.e. the Short Physical Performance Battery (SPPB) [7], and older age predicted discharge to a rehabilitation facility.

Geriatric Assessment and General Gastrointestinal Cancer Surgery

A few studies have analyzed various aspects of the GA in heterogeneous populations with GI malignancies. Because a GA may be considered unfeasible due to the time needed to complete the assessment, McCleary et al. [45] studied the feasibility of a computerbased GA in patients receiving treatment for GI malignancies. The majority of patients (97\%) were able to complete the GA using a touchscreen computer, and about half of the patients needed assistance. The GA added information to the clinical assessment for 75\% of patients at baseline. Badgwell et al. [46] sought to identify risk factors for adverse outcomes or increased resource utilization after abdominal cancer surgery in older patients. The study included 111 patients with a median age of 72 years, and the most common malignancies were CRC or HPB cancer. Independent predictors of discharge to nursing home were weight loss, ASA classification, and ECOG PS. Polypharmacy, defined as more than five medications, and weight loss predicted length of stay. Surprisingly, and contrary to most other studies in the literature across a variety of surgical procedures, no clinical or GA variables were associated with postoperative complications. $25 \%$ of patients were readmitted, but none of the preoperative GA or clinical variables predicted readmission. Unfortunately, the study did not include any objective measurements of physical performance. In comparison, a study from the USA that investigated the impact of a history of falls on postoperative complications in gastrointestinal surgery found that $100 \%$ of patients with three or more falls in the preceding 6 months experienced postoperative morbidity [47].

\section{Discussion}

Over the past 10 years, numerous studies have looked at the association between GA and outcomes for older patients with GI malignancies. Almost all studies show a clear association between elements of a GA and treatment outcomes. The evidence is particularly strong for CRC. For gastroesophageal and HPB cancer, the data is much more limited.

A few points are worth considering. It seems to be clear that frailty, defined as functional impairment, reduced mobility, or impairments in domains of the GA, is a consistently negative predictor of outcomes, in terms of both toxicity and survival. For CRC sur- gery, the majority of frail patients experience postoperative complications. However, as GI malignancies are lethal conditions and the malignant tumor often leads to complications, surgery will frequently be advised even though the risk of complications is high. In theory, a preoperative intervention targeted at the impairments identified through the GA may reduce postoperative morbidity or lead to less deterioration in postoperative functional status or quality of life. As we now know that GA predicts outcomes, future studies should investigate the effect of interventions based on the GA on various outcomes. Examples of pre-treatment interventions are:

(1) functional status: resistance training and aerobic training, prevention of falls;

(2) comorbidities and polypharmacy: critical appraisal of comorbidity by internal medicine specialist, optimization of medication regimen in relation to proposed treatment;

(3) nutritional status: dietary advice, prescription of supplements;

(4) depression or anxiety: cognitive therapy, medication if indicated;

(5) social support: arrange home nursing to help with medications, arrange meals.

In addition, because the GA provides a more comprehensive report of an older individual's health status, it may be a good starting point for discussing the goals of treatment to make sure that treatment is based on the patient's goals of care. Such a discussion may affect treatment decisions. Cancer treatment is often a trajectory consisting of surgery, sometimes several cycles of chemotherapy, and repeated appointments for radiotherapy. Unless we establish a baseline for functional and cognitive status before the patient embarks on that trajectory, we will not be able to detect functional and cognitive decline. Cancer specialists should be aware that a majority of older patients is not willing to accept severe cognitive and functional decline due to a treatment for life prolongation [48]. In order to increase our knowledge about cognitive and functional consequences of treatment and to better inform patients on what to expect, we need to include such endpoints in clinical trials. Thus, GA should not only be a pre-treatment concern in older patients with GI malignancies.

Although a GA encompasses several domains, one study showed that much of the information may be captured by self-report and even touchscreen computer technology [45]. Performing a physical performance measure such as 4-meter gait speed and a screening of cognitive impairment such as the Mini- $\operatorname{Cog}^{\mathrm{TM}}$ only takes a few extra minutes. The cancer specialist would benefit from learning to interpret the results of such assessments, and ideally be able to refer patients with cognitive impairment to a geriatrician or those patients with comorbidity and polypharmacy to a specialist in internal medicine. This is not always possible and depends on local resources.

\section{Conclusion}

Increasing evidence, particularly in $\mathrm{CRC}$, shows that elements from a GA and frailty are consistently associated with negative 
short- and long-term treatment outcomes in older patients with GI malignancies. Integration of GA into patient care will therefore add information about treatment tolerance in individual patients. In addition, a GA may identify unknown impairments, guide targeted interventions that may reduce the risk of adverse outcomes, establish a pre-treatment baseline for functional status, and provide information about life expectancy, and thus serve as a starting point for discussing the patient's treatment goals and priorities. Most of the GA can be done by self-report and should be inte- grated as a standard of care for older adults with GI malignancies. However, more research regarding the impact of geriatric interventions on traditional outcomes as well as patient-reported outcome measures is needed.

\section{Disclosure statement}

No conflicts of interest to disclose.

\section{References}

1 Wildiers H, Heeren P, Puts M, Topinkova E, JanssenHeijnen MLG, Extermann M, Falandry C, Artz A, Brain E, Colloca G, Flamaing J, Karnakis T, Kenis C, Audisio RA, Mohile S, Repetto L, Leeuwen BV, Milisen K, Hurria A: International Society of Geriatric Oncology consensus on geriatric assessment in older patients with cancer. J Clin Oncol 2014;32:2595-2603.

2 Huisingh-Scheetz M, Walston J: How should older adults with cancer be evaluated for frailty? J Geriatr Oncol 2017;8:8-15.

3 Mahoney FI, Barthel DW: Functional evaluation: the Barthel Index. Md State Med J 1965;14:61-65.

4 Lincoln NB, Gladman JR: The Extended Activities of Daily Living scale: a further validation. Disabil Rehabil 1992;14:41-43.

5 Cesari M, Kritchevsky SB, Penninx BW, Nicklas BJ, Simonsick EM, Newman AB, Tylavsky FA, Brach JS, Satterfield S, Bauer DC, Visser M, Rubin SM, Harris TB, Pahor M: Prognostic value of usual gait speed in well-functioning older people - results from the Health, Aging and Body Composition Study. J Am Geriatr Soc 2005;53:1675-1680.

6 Podsiadlo D, Richardson S: The timed 'Up \& Go': a test of basic functional mobility for frail elderly persons. J Am Geriatr Soc 1991;39:142-148.

7 Guralnik JM, Simonsick EM, Ferrucci L, Glynn RJ, Berkman LF, Blazer DG, Scherr PA, Wallace RB: A short physical performance battery assessing lower extremity function: association with self-reported disability and prediction of mortality and nursing home admission. J Gerontol 1994;49:M85-94.

8 Charlson ME, Pompei P, Ales KL, MacKenzie CR: A new method of classifying prognostic comorbidity in longitudinal studies: development and validation. J Chronic Dis 1987;40:373-383.

9 Miller MD, Paradis CF, Houck PR, Mazumdar S, Stack JA, Rifai AH, Mulsant B, Reynolds CF 3rd: Rating chronic medical illness burden in geropsychiatric practice and research: application of the Cumulative Illness Rating Scale. Psychiatry Res 1992;41:237-248.

10 Borson S, Scanlan JM, Chen P, Ganguli M: The MiniCog as a screen for dementia: validation in a populationbased sample. J Am Geriatr Soc 2003;51:1451-1454.

11 Nasreddine ZS, Phillips NA, Bedirian V, Charbonneau S, Whitehead V, Collin I, Cummings JL, Chertkow H: The Montreal Cognitive Assessment, MoCA: a brief screening tool for mild cognitive impairment. J Am Geriatr Soc 2005;53:695-699.

12 Guigoz Y: The Mini Nutritional Assessment (MNA) review of the literature - what does it tell us? J Nutr Health Aging 2006;10:466-485; discussion 485-487.

13 Yesavage JA, Brink TL, Rose TL, Lum O, Huang V, Adey M, Leirer VO: Development and validation of a geriatric depression screening scale: a preliminary report. J Psychiatr Res 1982;17:37-49.
14 Kristjansson SR, Nesbakken A, Jordhoy MS, Skovlund E, Audisio RA, Johannessen HO, Bakka A, Wyller TB: Comprehensive geriatric assessment can predict complications in elderly patients after elective surgery for colorectal cancer: a prospective observational cohort study. Crit Rev Oncol Hematol 2010;76:208-217.

15 Lee YH, Oh HK, Kim DW, Ihn MH, Kim JH, Son IT, Kang SI, Kim GI, Ahn S, Kang SB: Use of a comprehensive geriatric assessment to predict short-term postoperative outcome in elderly patients with colorectal cancer. Ann Coloproctol 2016;32:161-169.

16 Mokutani Y, Mizushima T, Yamasaki M, Rakugi H, Doki Y, Mori M: Prediction of postoperative complications following elective surgery in elderly patients with colorectal cancer using the Comprehensive Geriatric Assessment. Dig Surg 2016;33:470-477.

17 Reisinger KW, van Vugt JL, Tegels JJ, Snijders C, Hulsewe KW, Hoofwijk AG, Stoot JH, Von Meyenfeldt MF, Beets GL, Derikx JP, Poeze M: Functional compromise reflected by sarcopenia, frailty, and nutritional depletion predicts adverse postoperative outcome after colorectal cancer surgery. Ann Surg 2015;261:345-352.

18 Koroukian SM, Xu F, Bakaki PM, Diaz-Insua M, Towe TP, Owusu C: Comorbidities, functional limitations, and geriatric syndromes in relation to treatment and survival patterns among elders with colorectal cancer. J Gerontol Biol Sci Med Sci 2010;65:322-329.

19 Ommundsen N, Wyller TB, Nesbakken A, Jordhoy MS, Bakka A, Skovlund E, Rostoft S: Frailty is an independent predictor of survival in older patients with colorectal cancer. Oncologist 2014;19:1268-1275.

20 Ugolini G, Pasini F, Ghignone F, Zattoni D, Bacchi Reggiani ML, Parlanti D, Montroni I: How to select elderly colorectal cancer patients for surgery: a pilot study in an Italian academic medical center. Cancer Biol Med 2015;12:302-307.

21 Kristjansson SR, Jordhøy MS, Nesbakken A, Skovlund E, Bakka A, Johannessen H-O, Wyller TB: Which elements of a comprehensive geriatric assessment (CGA) predict post-operative complications and early mortality after colorectal cancer surgery? J Geriatr Oncol 2010;1: 57-65.

22 Rønning B, Wyller TB, Jordhøy MS, Nesbakken A, Bakka A, Seljeflot I, Kristjansson SR: Frailty indicators and functional status in older patients after colorectal cancer surgery. J Geriatr Oncol 2014;5:26-32.

23 Rønning B, Wyller TB, Nesbakken A, Skovlund E, Jordhøy MS, Bakka A, Rostoft S: Quality of life in older and frail patients after surgery for colorectal cancer - a follow-up study. J Geriatr Oncol 2016;7:195-200.

24 Tan KY, Kawamura YJ, Tokomitsu A, Tang T: Assessment for frailty is useful for predicting morbidity in elderly patients undergoing colorectal cancer resection whose comorbidities are already optimized. Am J Surg 2012;204:139-143.
25 Kristjansson SR, Rønning B, Hurria A, Skovlund E, Jordhøy MS, Nesbakken A, Wyller TB: A comparison of two pre-operative frailty measures in older surgical cancer patients. J Geriatric Oncol 2012;3:1-7.

26 Neuman HB, Weiss JM, Leverson G, O’Connor ES, Greenblatt DY, Loconte NK, Greenberg CC, Smith MA: Predictors of short-term postoperative survival after elective colectomy in colon cancer patients $\geq 80$ years of age. Ann Surg Oncol 2013;20:1427-1435.

27 Indrakusuma R, Dunker MS, Peetoom JJ, Schreurs WH: Evaluation of preoperative geriatric assessment of elderly patients with colorectal carcinoma. A retrospective study. Eur J Surg Oncol 2015;41:21-27.

28 Aaldriks AA, van der Geest LG, Giltay EJ, le Cessie S, Portielje JE, Tanis BC, Nortier JW, Maartense E: Frailty and malnutrition predictive of mortality risk in older patients with advanced colorectal cancer receiving chemotherapy. J Geriatr Oncol 2013;4:218-226.

29 Kroep JR, van Werkhoven E, Polee M, van Groeningen CJ, Beeker A, Erdkamp F, Weijl N, van Bochove A, Erjavec Z, Kapiteijn E, Stiggelbout AM, Nortier HW, Gelderblom H: Randomised study of tegafur-uracil plus leucovorin versus capecitabine as first-line therapy in elderly patients with advanced colorectal cancer TLC study. J Geriatr Oncol 2015;6:307-315.

30 Aparicio T, Jouve JL, Teillet L, Gargot D, Subtil F, Le Brun-Ly V, Cretin J, Locher C, Bouche O, Breysacher G, Charneau J, Seitz JF, Gasmi M, Stefani L, Ramdani M, Lecomte T, Mitry E: Geriatric factors predict chemotherapy feasibility: ancillary results of FFCD 2001-02 phase III study in first-line chemotherapy for metastatic colorectal cancer in elderly patients. J Clin Oncol 2013;31:1464-1470.

31 Aparicio T, Gargot D, Teillet L, et al: Geriatric factors analyses from FFCD 2001-02 phase III study of firstline chemotherapy for elderly metastatic colorectal cancer patients. Eur J Cancer 2017;74:98-108.

32 Ramsdale E, Polite B, Hemmerich J, Bylow K, Kindler HL, Mohile S, Dale W: The Vulnerable Elders Survey-13 predicts mortality in older adults with laterstage colorectal cancer receiving chemotherapy: a prospective pilot study. J Am Geriatr Soc 2013;61:20432044.

33 Decoster L, Vanacker L, Kenis C, Prenen H, Van Cutsem E, Van Der Auwera J, Van Eetvelde E, Van Puyvelde K, Flamaing J, Milisen K, Lobelle JP, De Greve J, Wildiers H: Relevance of geriatric assessment in older patients with colorectal cancer. Clin Colorectal Cancer 2016;DOI: 10.1016/j.clcc.2016.07.010.

34 Pujara D, Mansfield P, Ajani J, Blum M, Elimova E, Chiang YJ, Das P, Badgwell B: Comprehensive geriatric assessment in patients with gastric and gastroesophageal adenocarcinoma undergoing gastrectomy. J Surg Oncol 2015;112:883-887. 
35 Tegels JJ, de Maat MF, Hulsewé KW, Hoofwijk AG Stoot JH: Value of geriatric frailty and nutritional status assessment in predicting postoperative mortality in gastric cancer surgery. J Gastrointest Surg 2014;18: 439-445; discussion 445-446.

36 Yamana I, Takeno S, Shibata R, Shiwaku H, Maki K, Hashimoto T, Shiraishi T, Iwasaki A, Yamashita Y: Is the Geriatric Nutritional Risk Index a significant predictor of postoperative complications in patients with esophageal cancer undergoing esophagectomy? Eur Surg Res 2015;55:35-42.

37 Makiura D, Ono R, Inoue J, Kashiwa M, Oshikiri T, Nakamura T, Kakeji Y, Sakai Y, Miura Y: Preoperative sarcopenia is a predictor of postoperative pulmonary complications in esophageal cancer following esophagectomy: a retrospective cohort study. J Geriatr Oncol 2016;7:430-436.

38 Chen FF, Zhang FY, Zhou XY, Shen X, Yu Z, Zhuang CL: Role of frailty and nutritional status in predicting complications following total gastrectomy with D2 lymphadenectomy in patients with gastric cancer: a prospective study. Langenbecks Arch Surg 2016;401:813-822.

39 Yamamoto M, Yamasaki M, Sugimoto K, Maekawa Y, Miyazaki Y, Makino T, Takahashi T, Kurokawa Y, Nakajima K, Takiguchi S, Rakugi H, Mori M, Doki Y: Risk evaluation of postoperative delirium using comprehensive geriatric assessment in elderly patients with esophageal cancer. World J Surg 2016;40:2705-2712.
40 Meenen LC, Meenen DM, Rooij SE, Riet G: Risk prediction models for postoperative delirium: a systematic review and meta-analysis. J Am Geriatr Soc 2014;62: 2383-2390.

41 Fonck M, Brunet R, Becouarn Y, Legoux JL, Dauba J, Cany L, Smith D, Auby D, Terrebonne E, Traissac L, Mertens C, Soubeyran P, Bellera C, Rainfray M, Mathoulin-Pelissier S: Evaluation of efficacy and safety of FOLFIRI for elderly patients with gastric cancer: a first-line phase II study. Clin Res Hepatol Gastroenterol 2011;35:823-830

42 Kaibori M, Ishizaki M, Matsui $\mathrm{K}$, Iida $\mathrm{H}$, Inoue $\mathrm{K}$, Nagashima F, Kon M: Geriatric assessment as a predictor of postoperative complications in elderly patients with hepatocellular carcinoma. Langenbecks Arch Surg 2016;401:205-214.

43 Wagner D, Buttner S, Kim Y, Gani F, Xu L, Margonis GA, Amini N, Kamel IR, Pawlik TM: Clinical and morphometric parameters of frailty for prediction of mortality following hepatopancreaticobiliary surgery in the elderly. Br J Surg 2016;103:e83-92.

44 Dale W, Hemmerich J, Kamm A, Posner MC, Matthews JB, Rothman R, Palakodeti A, Roggin KK: Geriatric assessment improves prediction of surgical outcomes in older adults undergoing pancreaticoduodenectomy: a prospective cohort study. Ann Surg 2014; 259:960-965.
45 McCleary NJ, Wigler D, Berry D, Sato K, Abrams T, Chan J, Enzinger P, Ng K, Wolpin B, Schrag D, Fuchs CS, Hurria A, Meyerhardt JA: Feasibility of computerbased self-administered cancer-specific geriatric assessment in older patients with gastrointestinal malignancy. Oncologist 2013;18:64-72.

46 Badgwell B, Stanley J, Chang GJ, Katz MH, Lin HY, Ning J, Klimberg SV, Cormier JN: Comprehensive geriatric assessment of risk factors associated with adverse outcomes and resource utilization in cancer patients undergoing abdominal surgery. J Surg Oncol 2013;108:182-186

47 Jones TS, Dunn CL, Wu DS, Cleveland JC Jr, Kile D, Robinson TN: Relationship between asking an older adult about falls and surgical outcomes. JAMA Surg 2013;148:1132-1138.

48 Allore H, Fried TR, Bradley EH, Towle VR: Understanding the treatment preferences of seriously ill patients. N Engl J Med 2002;346:1061-1066. 